ARTICLE

DOI: $10.1038 / s 41467-018-04984-1$

\title{
Sensing of cytosolic LPS through caspy2 pyrin domain mediates noncanonical inflammasome activation in zebrafish
}

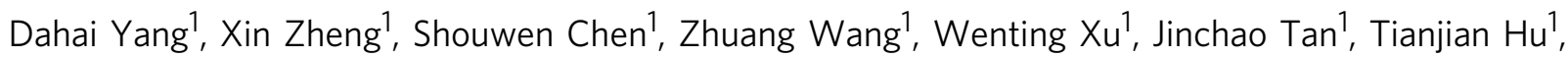
Mingyu Hou', Wenhui Wang ${ }^{1}$, Zhaoyan Gu1, Qiyao Wang1,2,3,4, Ruilin Zhang ${ }^{4,5}$, Yuanxing Zhang ${ }^{1,2,3,4} \&$ Qin Liu'1,2,3,4

The noncanonical inflammasome is critical for cytosolic sensing of Gram-negative pathogens. Here, we show that bacterial infection induces caspy2 activation in zebrafish fibroblasts, which mediates pyroptosis via a caspase-5-like activity. Zebrafish caspy2 binds directly to lipopolysaccharide via the $\mathrm{N}$-terminal pyrin death domain, resulting in caspy2 oligomerization, which is critical for pyroptosis. Furthermore, we show that caspy 2 is highly expressed in the zebrafish gut and is activated during infection. Knockdown of caspy 2 expression impairs the ability of zebrafish to restrict bacterial invasion in vivo, and protects larvae from lethal sepsis. Collectively, our results identify a crucial event in the evolution of pattern recognition into the death domain superfamily-mediated intracellular lipopolysaccharide-sensing pathway in innate immunity.

\footnotetext{
${ }^{1}$ State Key Laboratory of Bioreactor Engineering, East China University of Science and Technology, Shanghai 200237, China. ${ }^{2}$ Laboratory for Marine Biology and Biotechnology, Qingdao National Laboratory for Marine Science and Technology, Qingdao 266071, China. ${ }^{3}$ Shanghai Engineering Research Center of Marine Cultured Animal Vaccines, Shanghai 200237, China. ${ }^{4}$ Shanghai Collaborative Innovation Center for Biomanufacturing, Shanghai 200237, China. ${ }^{5}$ State Key Laboratory of Genetic Engineering, School of Life Sciences, Fudan University, Shanghai 200433, China. Correspondence and requests for materials should be addressed to Q.L. (email: qinliu@ecust.edu.cn)
} 
T he noncanonical inflammasome is critical for the cytosolic sensing of several Gram-negative pathogens, and its discovery might expand the spectrum of known pyroptosis mediators in mammals ${ }^{1-5}$. In humans, caspase- 4 and caspase-5 seem to function similarly to mouse caspase-11, and are activated by intracellular lipopolysaccharide (LPS) to induce gasdermin D cleavage-triggered cell pyroptosis ${ }^{5-7}$, which has proinflammatory effects and mediates innate immune defense against a variety of pathogens. Given the important function of the noncanonical inflammasome in mammalian immune systems, understanding related signaling pathway in lower vertebrates is critical.

The zebrafish is an important vertebrate organism to study host-pathogen interactions and immunity ${ }^{8}$. To date, at least five functional caspases have been identified in zebrafish, including the inflammatory caspy (also named as caspase-A), caspy2 (also named as caspase- $\mathrm{B}$ ), and caspase- $\mathrm{C}^{9-11}$. The zebrafish genome contains caspase- $\mathrm{C}$ and a caspase-C-like protease, which have highest similarity to human caspase-4; however, these caspase variants do not have apoptotic protein domains in their N-terminal regions ${ }^{9}$. In addition, the catalytic domains of zebrafish caspy and caspy 2 have highest homology with those of human caspase- 1 and caspase-5, with sequence similarity of 54 and $57 \%$, respectively ${ }^{9-11}$. Interestingly, both caspy and caspy 2 have a pyrin domain at their $\mathrm{N}$-terminal instead of the typical caspase activation and recruitment domain (CARD) of inflammatory caspases ${ }^{9-11}$. Compared with the identified role of caspy in mediating canonical inflammasome activation signaling $11-14$, the biological function of caspy 2 is unclear. Previous work has shown that caspy2 has the highest homology to human caspase-4/5, but cannot interact with apoptosis-associated speck-like containing a CARD (ASC) ${ }^{10}$. Enzyme activity analysis showed that caspy2 has a preferred caspase- 5 substrate specificity ${ }^{10}$. Moreover, caspy2-transfected mammalian cells have morphological features of adherent cells undergoing apoptosis, such as rounding and membrane blebbing ${ }^{10,15}$. However, whether the zebrafish caspy2 binds cytosolic LPS and induces noncanonical inflammasome mediated pyroptosis in lower vertebrates is unclear.

Here, we report the functional identification and characterization of zebrafish caspy2, and provide evidence that its $\mathrm{N}$ terminal pyrin death domain (PYD) is critical for LPS binding, and responsible for noncanonical inflammasome activation in zebrafish nonmyeloid cells. In addition, we show that caspy 2 is highly expressed in the gut, and has an essential function in restricting bacterial infection in intestinal sites. Moreover, knockdown of caspy 2 protects larvae from lethal sepsis. Our study first establishes the existence of caspy2-mediated noncanonical inflammasome activation in zebrafish, and clarifies the role of caspy2 activation in promoting host defense, which provides important insight into the evolution of pattern recognition in the death domain superfamily-mediated intracellular LPS-sensing pathway for innate immunity.

\section{Results}

Caspase- 5 activity-mediated pyroptosis in zebrafish fibroblasts. To analyze pyroptosis in zebrafish cells, we infected zebrafish fibroblasts (ZF4) with 0909I Edwardsiella piscicida, a hemolysinoverexpressing strain that specifically promotes caspase-4- and gasdermin D-gated noncanonical inflammasome activationdependent cell death in mammalian HeLa cells (Supplementary Fig. 1). ZF4 cells exhibited pyroptotic morphological features, such as cell swelling, membrane rupture (as shown by propidium iodide (PI)-positive staining, Supplementary Fig. 2), and significant cytotoxicity (Fig. 1a) following infection with 0909I $E$. piscicida. The morphology of ZF4 cells infected with wild-type $E$. piscicida (EIB202) was similar to that of untreated cells (Supplementary Fig. 2).

To investigate the link between pyroptosis and caspase activity in zebrafish fibroblasts, we assessed the ability of cell extracts to cleave a panel of fluorogenic and chromogenic peptide substrates of mammalian caspases. Notably, extracts from 0909I E. piscicidainfected ZF4 cells preferentially cleaved the fluorogenic substrate of human caspase-5, Ac-Trp-Glu-His-Asp-7-amino-4-methylcoumarin (Ac-WEHD-AFC), but did not cleave the other caspase substrates tested (Fig. 1b). The cytotoxicity and pyroptotic morphology induced by 0909I E. piscicida were effectively inhibited by Z-WEHD-FMK and Z-VAD-FMK, which are caspase-5 and pan-caspase inhibitors, respectively (Fig. 1c, d). In contrast, Z-YVAD-FMK, Ac-DEVD-CHO, and Ac-LEVDCHO, which inhibit caspase-1, caspase-3/7, and caspase-4, respectively, did not restrict the cytotoxicity or pyroptotic morphological changes in 0909I E. piscicida-infected ZF4 cells (Fig. 1c, d). Thus, these results indicated that the induction of pyroptotic morphology by 0909I E. piscicida in zebrafish fibroblasts is dependent on a caspase-5-like activity.

To further confirm the noncanonical inflammasome activation exists in zebrafish fibroblasts, we primed ZF4 cells with Pam3CSK4 and then stimulated them with cholera toxin B subunit (CTB) plus LPS, a commonly used stimulus that activates the noncanonical inflammasome. Consistent with 0909I E. piscicida infection, ZF4 cells exhibited significant cytotoxicity (Fig. 1e). The cytotoxicity induced by cytosolic LPS delivery was effectively inhibited by Z-WEHD-FMK and Z-VAD-FMK, but not Z-YVAD-FMK, Ac-DEVD-CHO, and Ac-LEVD-CHO (Fig. 1f). Moreover, lysates of LPS-transfected ZF4 cells showed significant preferential cleavage of Ac-WEHD-AFC, but did not cleave the other caspase substrates tested (Fig. 1g). Taken together, these results suggested that the noncanonical inflammasome is present in zebrafish.

According to previous research, both caspy and caspy 2 are active caspases with different substrate specificity. Caspy preferentially cleaved Ac-YVAD-AMC, a caspase- 1 substrate, whereas caspy2 was more active on Ac-WEHD-AFC, a preferred substrate of caspase- $5^{10,11}$. Based on our results above, the pyroptosis induced in ZF4 cells is dependent on caspase-5-like activity, thus, we expect that caspy 2 is an enzymatically active caspase in zebrafish, which might responsible for noncanonical inflammasome activation. As a more direct test of caspy2 activity, a panel of fluorogenic and chromogenic peptide substrates of mammalian caspases were incubated with caspy 2 immunoprecipitated from extracts of cells transfected with a caspy2-expressing plasmid. Notably, caspy2 activity was highest with Ac-WEHDAFC, a preferred substrate of caspase-5, and it did not cleave the other substrates (Supplementary Fig. 3a). Determination of the substrate-binding specificity of caspy2 to Ac-WEHD-AFC showed that this enzyme exhibited strong substrate concentration-dependent activity (Supplementary Fig. 3b). Furthermore, two caspy 2 mutants, one carrying a Ser substitution of the critical Cys residue at position 296 (C296A, indicated in Supplementary Fig. 4) and another with an N-terminal pyrin truncation $(\triangle \mathrm{PYD}$, indicated in Supplementary Fig. 4), demonstrated impaired substrate-binding activity (Supplementary Fig. 3b). These resuslts suggested that zebrafish caspy2 is an enzymatically active caspase with caspase-5-like substrate specificity, which might play an important role in inducing pyroptosis in zebrafish fibroblasts.

Caspy2 activation is critical for bacterial infection-induced pyroptosis. To further validate the role of caspy 2 in 0909I $E$. piscicida infection-induced pyroptosis in zebrafish fibroblasts, the 

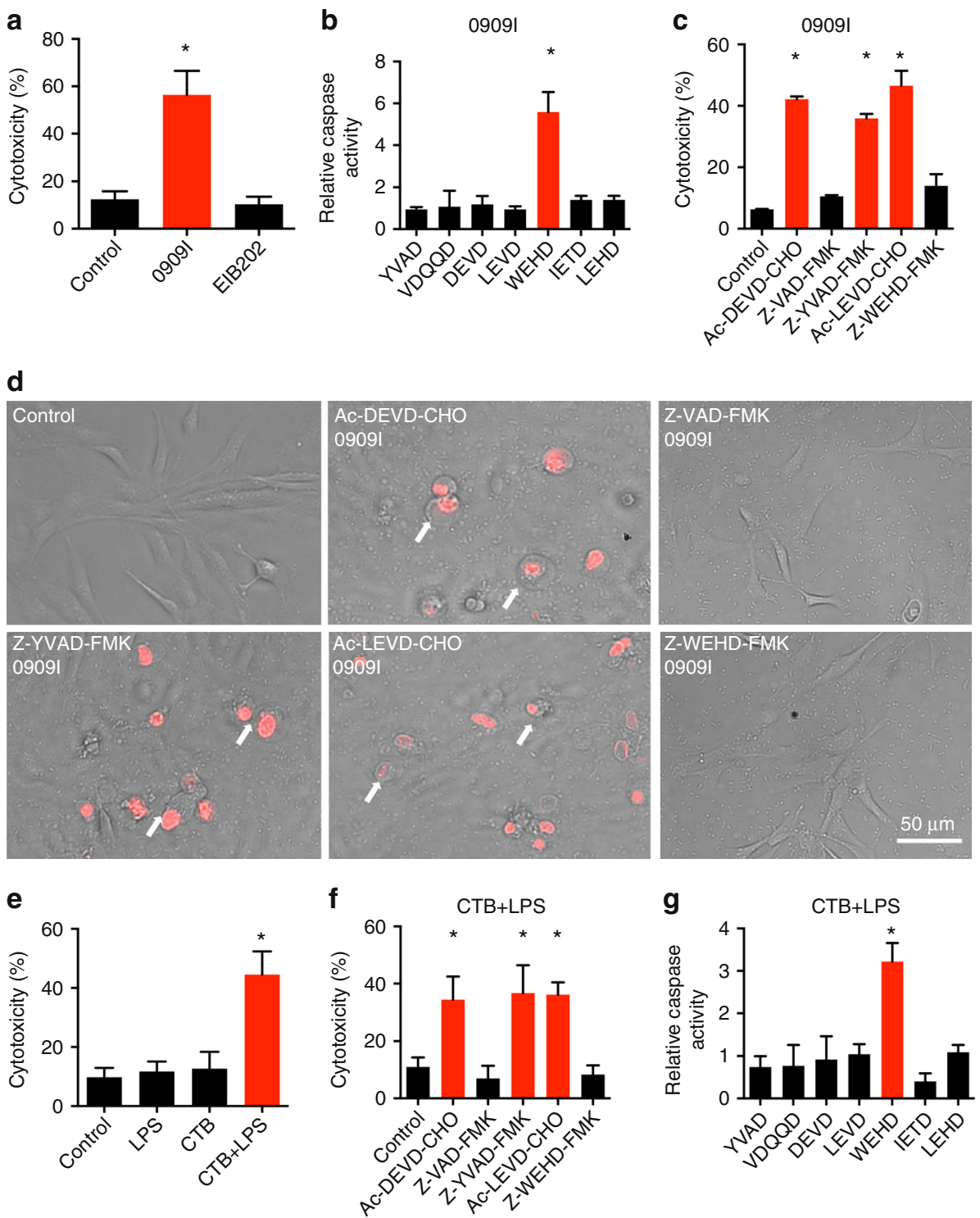

Fig. 1 Caspase-5-like activity is essential for pyroptosis in zebrafish fibroblasts. a ZF4 zebrafish fibroblasts were infected with wild-type (ElB202) or 0909| E. piscicida for $2 \mathrm{~h}$ at a multiplicity of infection (MOI) of 50, or left uninfected. Supernatants from the indicated ZF4 cells were analyzed for cell death, as measured by lactate dehydrogenase (LDH) release. b ZF4 cells were infected with $0909 \mathrm{I}$. piscicida for $2 \mathrm{~h}$ at an MOI of 50 . Relative caspase activity was then measured by incubating cell lysates with fluorogenic and chromogenic substrates of caspase-1 (YVAD), caspase-2 (VDQQD), caspase-3/7 (DEVD), caspase-4 (LEVD), caspase-5 (WEHD), caspase-8 (IETD), and caspase-9 (LEHD). c, d ZF4 cells were treated with caspase-3/7, pan-caspase, caspase-1, caspase-4, and caspase-5 inhibitors (Ac-DEVD-CHO, Z-VAD-FMK, Z-YVAD-FMK, Ac-LEVD-CHO, and Z-WEHD-FMK, respectively). c LDH release for cell death was measured $2 \mathrm{~h}$ after 0909I E. piscicida infection. $\mathbf{d}$ Images were taken after 0909l E. piscicida infection for $2 \mathrm{~h}$. Propidium iodide (PI) was added to detect the loss of plasma membrane integrity. Arrows indicate cells exhibiting pyroptotic-like features. Scale bar, $50 \mu \mathrm{m}$. e-g ZF4 cells were primed with Pam3CSK 4 for $4 \mathrm{~h}$, before being stimulated with cholera toxin B subunit (CTB) plus LPS, LPS, or CTB alone for $12 \mathrm{~h}$. e Supernatants from the indicated ZF4 cells were analyzed for cell death, as measured by lactate dehydrogenase (LDH) release. $\mathbf{f}$ Cytosolic LPS-delivered ZF4 cells were treated with indicated caspase inhibitors as in Fig. 1c, Supernatants from the indicated ZF4 cells were analyzed for cell death, as measured by lactate dehydrogenase $(\mathrm{LDH})$ release. $\mathbf{g}$ Relative caspases activity was measured by incubating cell lysates with indicated fluorogenic and chromogenic substrates as in Fig. $1 \mathrm{~b}$. a-g Results are representative of at least three independent experiments, and error bars denote the SD of triplicate wells. ${ }^{*} p<0.05$ ( $t$ test)

CRISPR/Cas9 genome-editing tool was applied to knockout (KO) caspy2 in ZF4 cells, using a caspy2-specific guide RNA (gRNA) (Supplementary Fig. 5). The pSpCas9(BB)-caspy2 gRNA-green flourescent plasmid (GFP) plasmids were used at $1 \mu \mathrm{g} / \mathrm{ml}$ and resulted in disruption of the caspy 2 locus of transfected ZF4 cells (Supplementary Fig. 5). Importantly, caspy2 deficiency impaired the development of the pyroptotic morphology induced by 0909I E. piscicida infection (Fig. 2a), and prevented the associated cytotoxicity (Fig. 2b). Moreover, when we infected wild-type and caspy2-KO ZF4 cells with wild-type (EIB202) or 0909I E. piscicida, we observed a comparable transcriptional induction of caspy2 (Fig. 2c); however, the cleavage of procaspy2, as determined by immunoblotting using an antibody that recognizes p20, the cleaved form of caspy2, was only detected during 0909I E. piscicida infection (Fig. 2c). Taken together, these findings indicated that the existence of pyroptosis in zebrafish is mediated by caspy 2 activation during 0909I E. piscicida infection. 
a
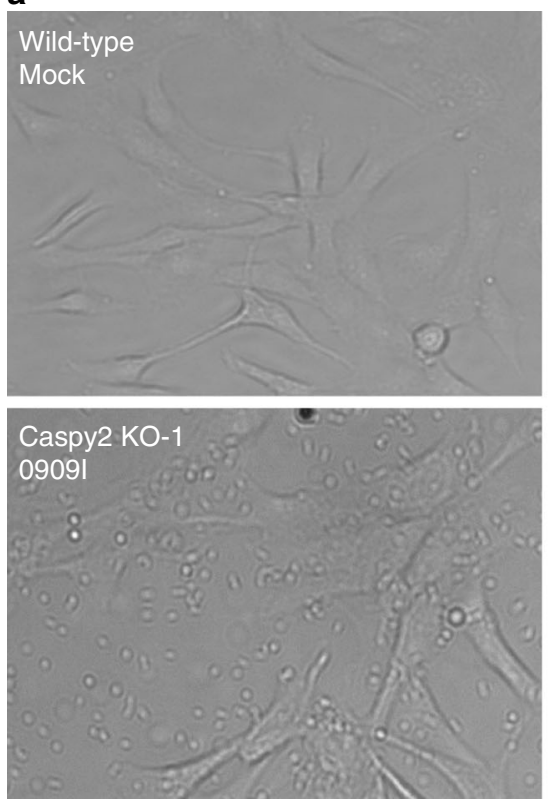

b

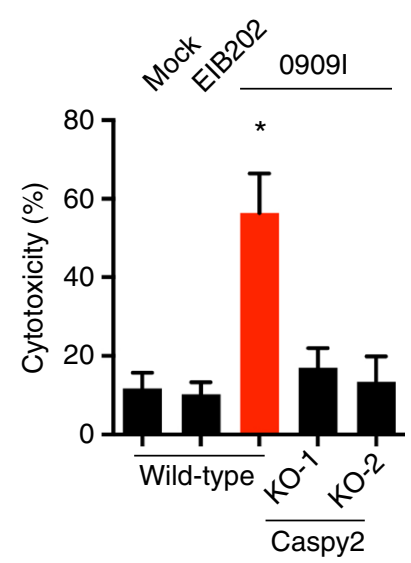

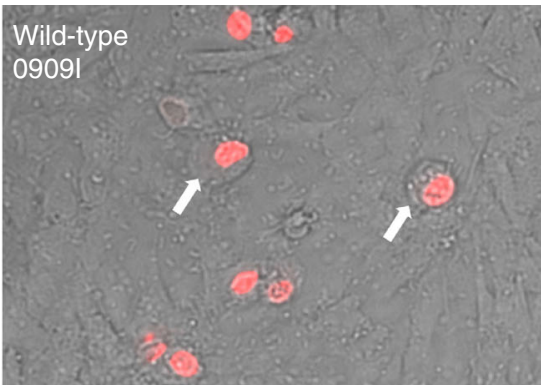

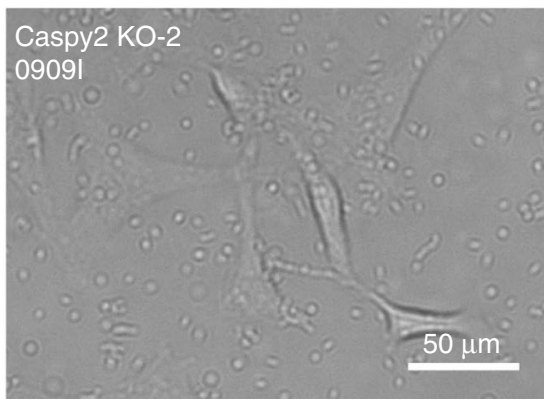

C

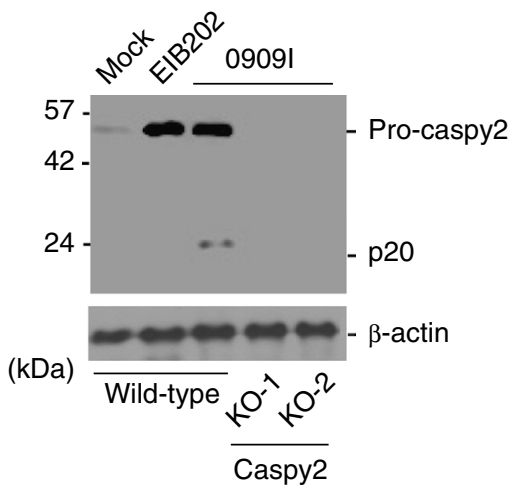

Fig. 2 Caspy2 plays a critical role in pyroptosis in zebrafish fibroblasts. a-c Wild-type and caspy2-knockout (KO) ZF4 cells were infected with ElB2O2 or $0909 \mathrm{I}$. piscicida for $2 \mathrm{~h}$ at an multiplicity of infection (MOI) of 50, or left uninfected. a Images were taken as in Fig. 1d. Propidium iodide (PI) was added to detect the loss of plasma membrane integrity. Arrows signify cells with pyroptotic-like features. Scale bar, $50 \mu \mathrm{m}$. $\mathbf{b}$ Supernatants from the indicated ZF4 cells were analyzed for cell death measured by lactate dehydrogenase (LDH) release. c Mixtures of cell lysates and supernatants were subjected to immunoblotting. (a-c) Results are representative of at least three independent experiments, and error bars denote the SD of triplicate wells. ${ }^{*} p<0.05$ ( $t$ test)

Caspy 2 binds directly to LPS and undergoes oligomerization. The N-terminal death domain superfamily (caspase-recruitment domain, CARD) of caspase-4/5 and caspase-11 can bind LPS and activate the noncanonical inflammasome ${ }^{6}$. However, the $\mathrm{N}$ terminal domain of caspy2 is most homologous to that of human Cryopyrin/PYPAF1 (46\% similarity), a pyrin domain containing Nod-family protein ${ }^{10}$. Sequence analysis also showed that the pyrin domain of caspy 2 exhibited significant homology not only to pyrin domains, but also to the CARDs of Xenopus caspase-1 (49\% similarity) and bovine caspase-13 (45\% similarity, previously known as human caspase-13) ${ }^{10}$. Thus, we speculated that the pyrin domain superfamily might possess a similar function in directly binding LPS. To test this idea, we carried out a series of pulldown assays to test the LPS-binding affinity and specificity of caspy2 and caspy. Biotinylated-ultrapure LPS efficiently precipitated caspy2, caspy2 (C296A) and caspy2 PYD, but not caspy2 $(\triangle \mathrm{PYD})$, caspy or caspy PYD (Fig. 3a). However, neither caspy nor caspy2 could be pulled down by biotinylatedPam3CSK4, another immunostimulatory peptide from bacterial lipoprotein (Fig. 3a). In addition, in enzyme linked immunosorbent assays (ELISAs) of LPS-binding activity. Similar to caspase11, caspy2, caspy2 (C296A), and caspy2 PYD showed strong affinity to immobilized LPS, with the number of LPS-caspy2 complexes increasing in a concentration-dependent manner (Supplementary Fig. 6). However, caspy2 ( $\triangle \mathrm{PYD}$ ), caspy, caspy PYD, and caspase-1 displayed no affinity for LPS (Supplementary Fig. 6), demonstrating that the caspy $2 \mathrm{~N}$-terminal pyrin domain is crucial for LPS recognition in zebrafish (Supplementary Fig. 6). Consistently, neither caspy nor caspy 2 were not precipitated by biotinylated-Pam3CSK4 (Fig. 3a).

Recent studies have shown that caspase-4/5 and caspase-11 undergoes oligomerization upon LPS binding, which is required for the caspases' catalytic activity ${ }^{6}$. Consequently, we used $0.5 \%$ Triton X-100 to reduce the LPS-induced caspases into a smaller homogenous form on blue native polyacrylamide gel electrophoresis (PAGE) ${ }^{6}$, followed by western blotting with anti-HA antibodies. We found that purified HA-tagged caspy2 and caspy2 PYD from transfected HEK293T cell were oligomerized upon 
a
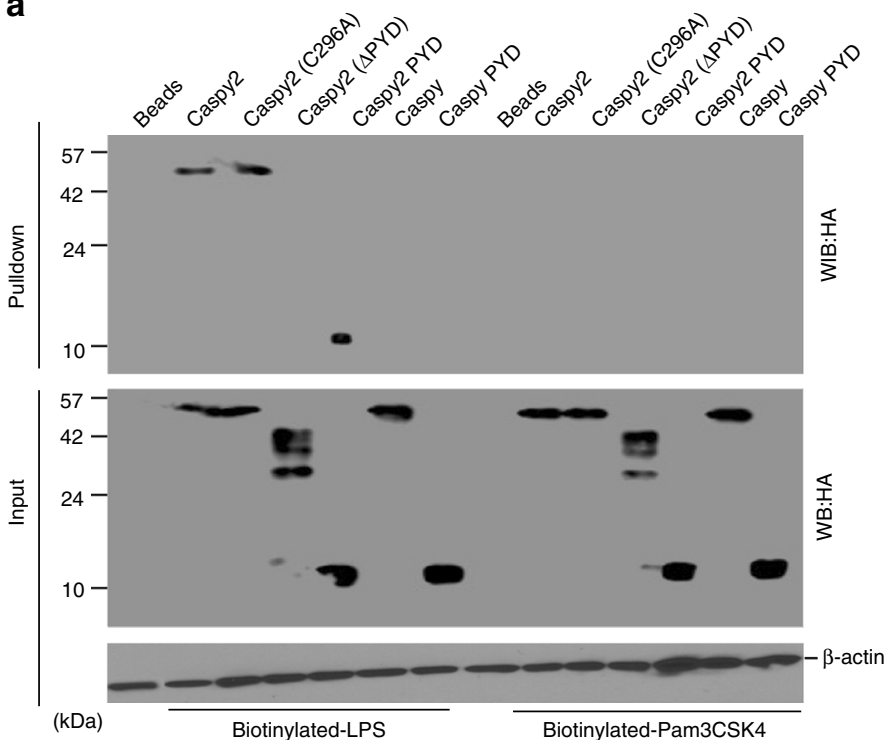

C

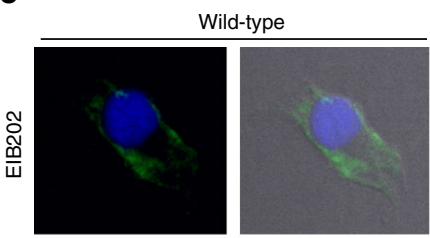

$0.00 \pm 0.00 \%$

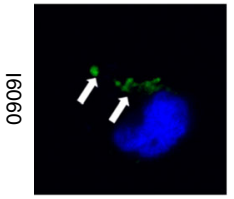

$32.84 \pm 3.76 \%$

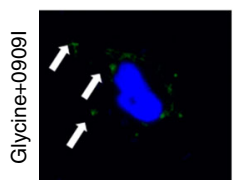

$44.52 \pm 4.93 \%$
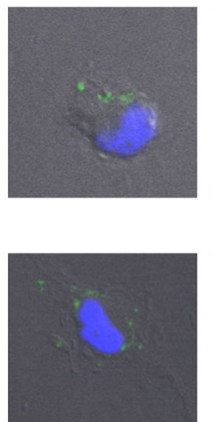

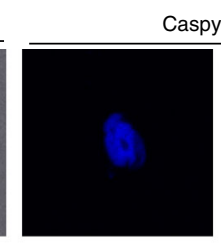

$0.00 \pm 0.00 \%$

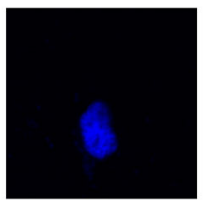

$0.00 \pm 0.00 \%$

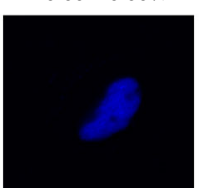

$0.00 \pm 0.00 \%$ b

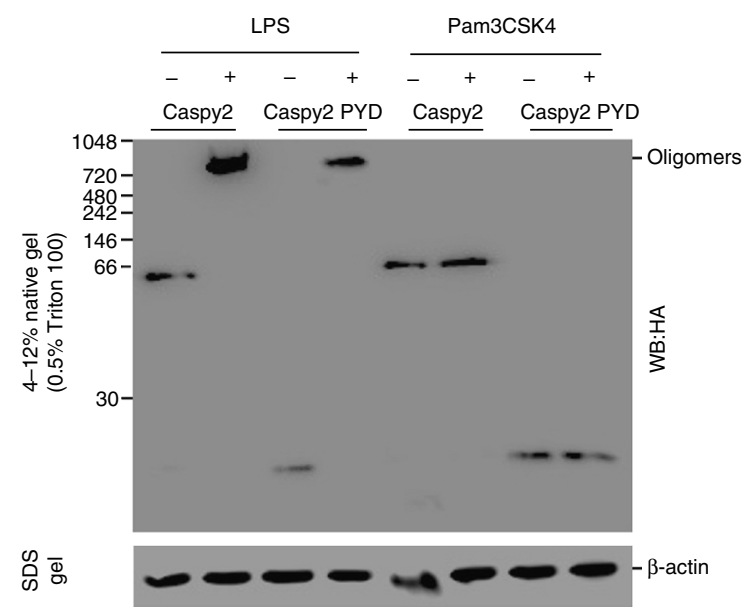

d

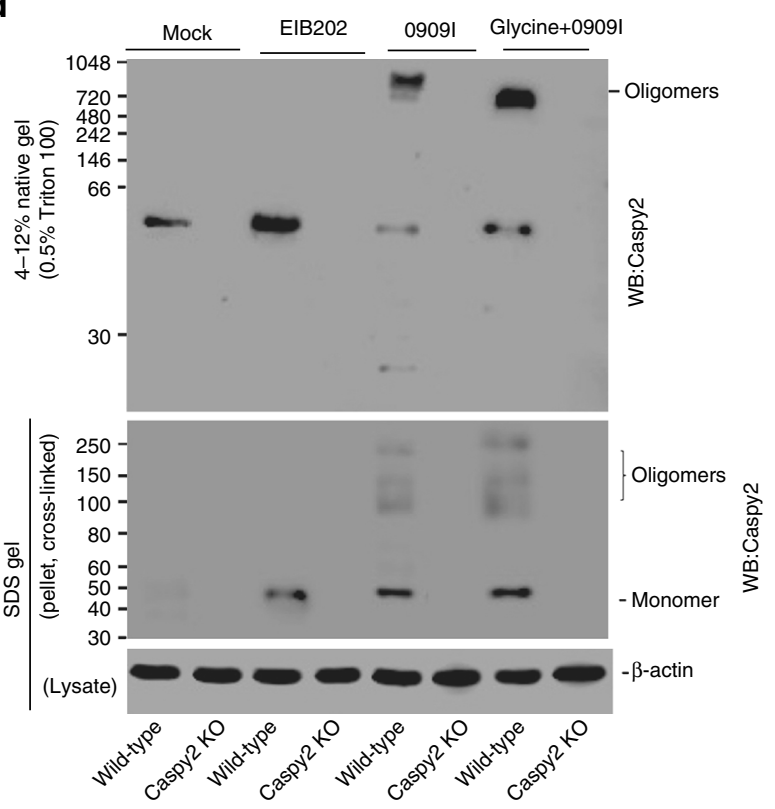

Fig. 3 Caspy2 directly binds to LPS to induce its oligomerization. a Streptavidin pulldown assays of the binding of biotin-conjugated LPS and Pam3CSK4 to purified HA-tagged caspy2, caspy2 (C296A), caspy2 ( PYD), caspy2 PYD, caspy and caspy PYD in transfected HEK293T cells. Shown are anti-HA immunoblots of pulled down proteins and total lysates (input). b Purified HA-tagged caspy2 and caspy2 PYD in transfected HEK293T cells were incubated with LPS or Pam3CSK4. The samples were analyzed by the pore-limited native gel electrophoresis. c Wild-type and caspy2-KO ZF4 cells were infected with wild-type (EIB202) or 0909l E. piscicida (with/without $10 \mathrm{mM}$ glycine) for $2 \mathrm{~h}$ at a multiplicity of infection (MOI) of 50. Confocal laser scanning microscopic analysis of nuclei (2-(4-amidinophenyl)-1H-indole-6-carboxamidine (DAPI), blue) and caspy2 foci (green, white arrowheads). Direct interference contrast (DIC)/phase images are shown. Scale bar, $10 \mu \mathrm{m}$. Statistics of the percentages of cells showing signals for caspy 2 foci are listed below. (Approximately 200 cells were counted in each sample. Mean \pm SD of triplicate samples.) d Wild-type and caspy2-KO ZF4 cells were infected with wild-type (EIB202) or 0909I E. piscicida (with/without $10 \mathrm{mM}$ glycine) for $2 \mathrm{~h}$ at an MOI of 50, or left untreated (Mock). The samples were analyzed by the pore-limited native gel electrophoresis and immunoblotting (upper panel). Cell lysates and DSS cross-linked pellets from wild-type and caspy2-KO ZF4 cells treated as indicated were analyzed by immunoblotting for caspy2 oligomerization (lower panel). WB western blot. Results are representative of at least three independent experiments

incubation with LPS, but not with Pam3CSK4 (Fig. 3b). Furthermore, to detect whether the caspy2 oligomerization occurs during bacterial infection, we utilized an antibody that recognizes the caspy2 $\mathrm{N}$-terminal PYD to probe the caspy 2 foci. A significantly higher quantity of caspy2 foci was observed in 0909I E. piscicida-infected wild-type ZF4 cells, compared with that in EIB202-infected cells (Fig. 3c), and no signaling was detected in caspy2-KO ZF4 cells infected with either bacterial strains (Fig. 3c).
Moreover, we also used the cytoprotectant glycine to inhibit pyroptotic cell lysis during 0909I E. piscicida infection, and observed more caspy 2 foci in 0909I E. piscicida-infected wild-type ZF4 cells (Fig. 3c). Consistently, caspy 2 treated with cross-linking agents remained as oligomers on the sodium dodecyl sulfate (SDS) gel, and oligomerization was triggered by infection with 0909I, but not with EIB202 E. piscicida (Fig. 3d). In addition, to assess caspy 2 assembly during infection, wild-type or caspy2-KO 
a

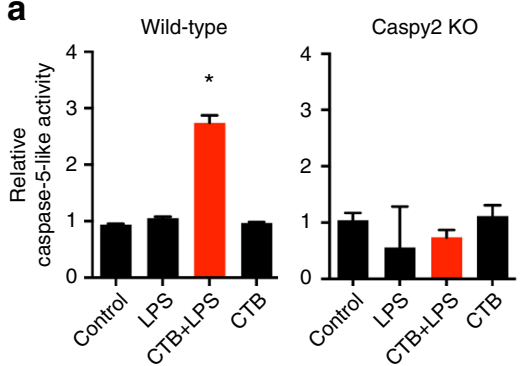

d

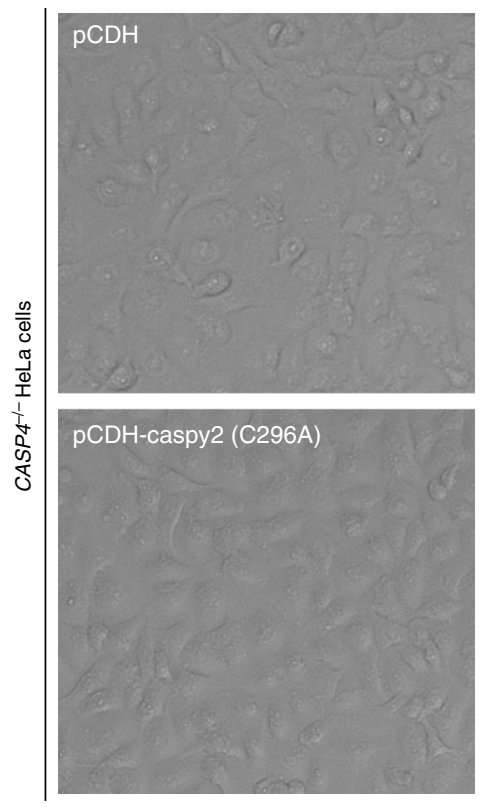

b
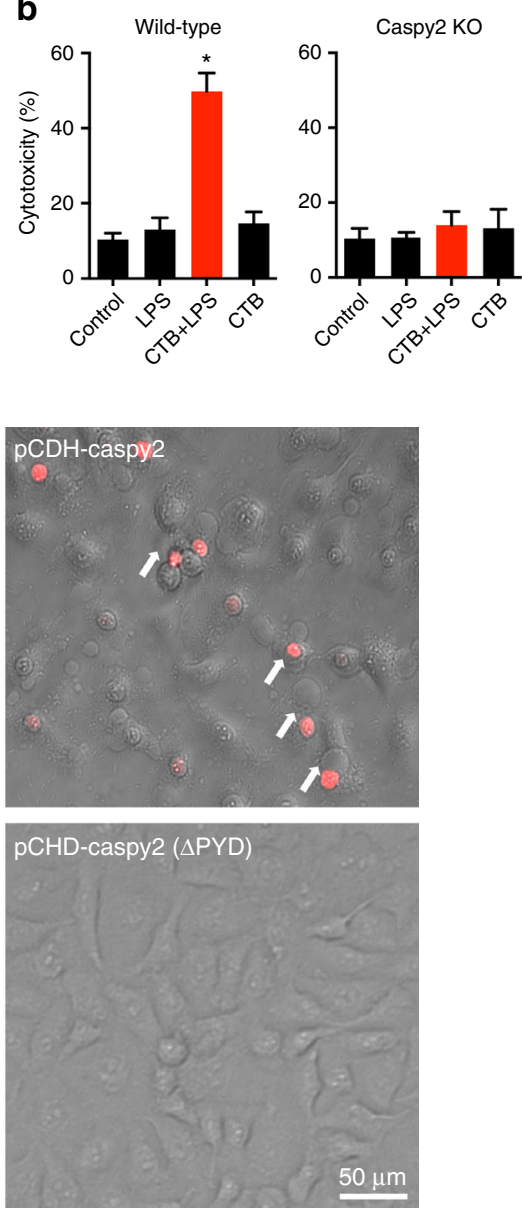

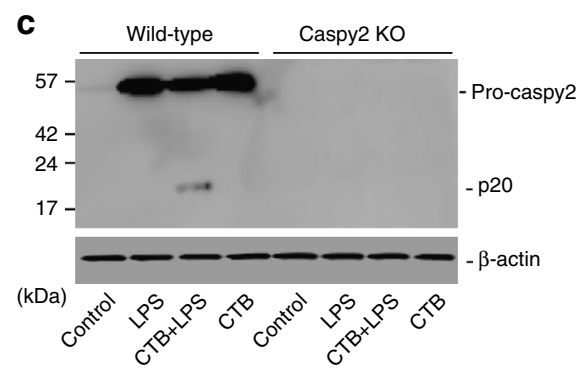

e
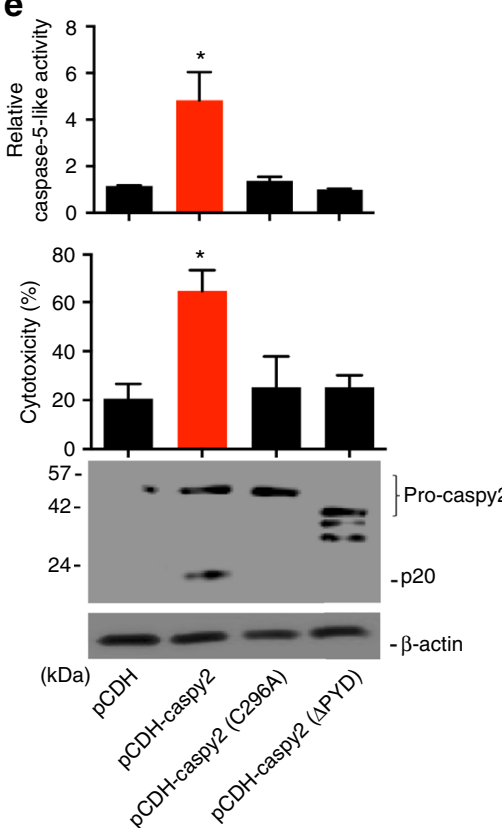

Fig. 4 Intracellular LPS-triggered-caspy2 noncanonical inflammasome activation. a-c ZF4 cells were primed with Pam3CSK4 for $4 \mathrm{~h}$, before being stimulated with cholera toxin B subunit (CTB) plus LPS, LPS, or CTB alone for $12 \mathrm{~h}$. Relative caspase-5-like activity was measured by incubating cell lysates with fluorogenic and chromogenic caspase-5 substrates (WEHD) (a). Supernatants from the indicated HeLa cells were analyzed for cell death, as measured by lactate dehydrogenase (LDH) release (b). Mixtures of cell lysates and supernatants were subjected to immunoblotting (c). d, e CASP4-/HeLa cells were transduced with a vector expressing wild-type caspy2, caspy2 (C296A), caspy2 ( $P$ PYD), or the empty vector. Cells were primed with LPS for $4 \mathrm{~h}$, before being stimulated with CTB plus LPS for $12 \mathrm{~h}$. Images were taken as in Fig. 1d (d). Propidium iodide (PI) was added to detect the loss of plasma membrane integrity. Arrows denote cells with pyroptotic-like features. Scale bar, $50 \mu \mathrm{m}$. Relative caspase-5-like activity and LDH release assays were conducted as in $\mathrm{a}$ and $\mathrm{b}(\mathbf{e})$. Immunoblotting for the caspy 2 forms indicated is shown. a-e Results are representative of at least three independent experiments, and error bars denote the SD of triplicate wells. ${ }^{\star} p<0.05$ ( $t$ test)

ZF4 cells were stimulated with 0909I or EIB202 E. piscicida, and digitonin-solublized cell lysates were resolved by blue native PAGE with $0.5 \%$ Triton X-100, followed by western blotting with anti-caspy2 antibodies. A large oligomeric complex containing caspy 2 was induced in wild-type ZF4 cells upon infection with 0909I, but not with EIB202 E. piscicida (Fig. 3d). Taken together, these results suggested that zebrafish caspy2 directly binds LPS and forms oligomers responding to bacterial infection.

Intracellular LPS stimulates caspy 2 activation. To dissect the cytoplasmic sensing of LPS by caspy2, we primed wild-type and caspy2-KO ZF4 cells with Pam3CSK4 and then stimulated them with CTB plus LPS, a commonly used stimulus that activates the noncanonical inflammasome. Lysates of wild-type, but not caspy2-KO, LPS-transfected ZF4 cells showed significant preferential cleavage of Ac-WEHD-AFC (Fig. 4a). Notably, caspy2 was important for intracellular LPS-induced cytotoxicity (Fig. 4b), and the formation of the p20 cleaved form of caspy 2 was detected in wild-type LPS-transfected ZF4 cells (Fig. 4c). These results indicated that caspy 2 is essential for intracellular
LPS-triggered noncanonical inflammasome activation in zebrafish.

To determine whether the N-terminal pyrin domain or the catalytic activity of caspy2 is required for pyroptosis, we expressed the wild-type, C296A, or $\triangle \mathrm{PYD}$ form of this enzyme in CASP4 ${ }^{-1}$ HeLa cells (Fig. 4d, e). Importantly, wild-type caspy2, but not the C296A or $\triangle \mathrm{PYD}$ mutants, restored intracellular LPS-induced pyroptosis in these cells (Fig. 4d, e). In addition, we expressed the wild-type, C254S, or $\triangle$ CARD form of caspase-11 in CASP4 ${ }^{-/-} \mathrm{HeLa}$ cells. Consistently, wild-type caspase-11, but not the C254S or $\triangle$ CARD mutants, restored intracellular LPS-induced pyroptosis in these cells (Supplementary Fig. 7). These observations suggested that the zebrafish caspy 2 pyrin domain has a function similar to that of the caspase11 CARD domain in recognizing intracellular LPS to mediate its oligomerization (Supplementary Fig. 8); in addition, the catalytic activity is required for pyroptosis in zebrafish.

Caspy2 is highly expressed in developmental gut. A previous study suggested that although caspy 2 is expressed during 
a

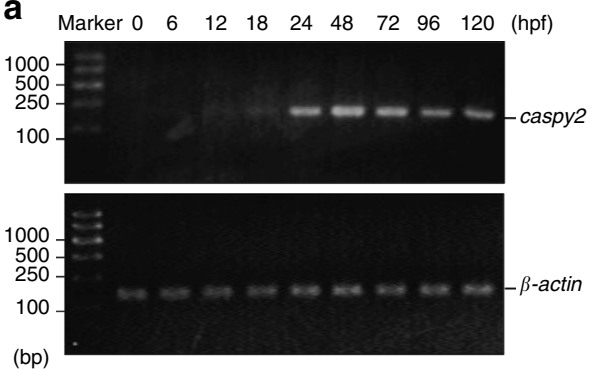

C

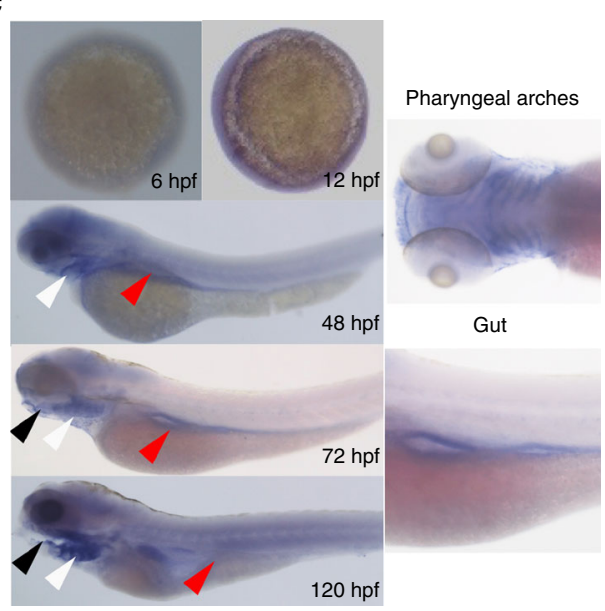

b

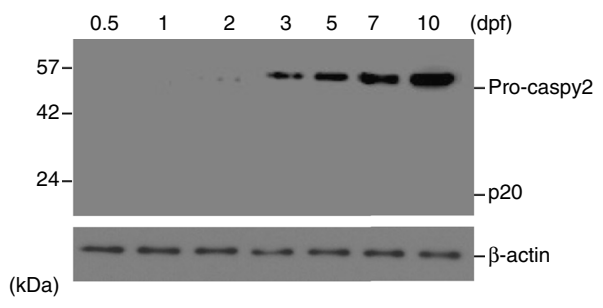

d

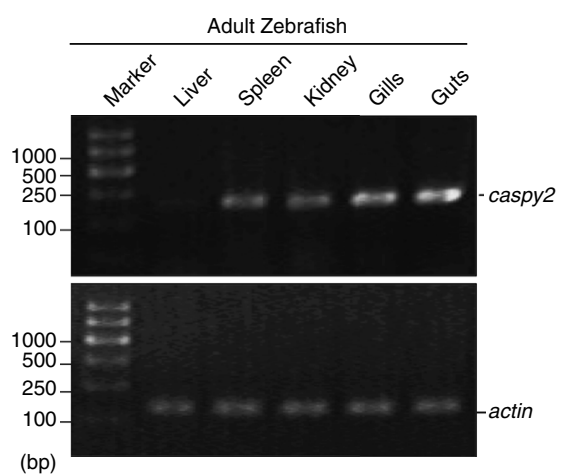

Fig. 5 Pattern of caspy2 expression in zebrafish. a Reverse transcription polymerase chain reaction (RT-PCR) analysis of relative caspy2 expression at various stages of development. $\mathbf{b}$ Immunoblotting analysis of caspy2 levels at various stages of whole larvae development. c Whole-mount in situ hybridization targeting caspy 2 mRNA in zebrafish at the developmental stages indicated. Black arrowheads, mouth; white arrowheads, pharyngeal arches; red arrowheads, gut. d RT-PCR analysis of relative caspy2 expression in the indicated adult zebrafish organs. a-d Results are representative of at least three independent experiments

zebrafish development, such expression is largely limited to the epidermis, mouth, and pharyngeal arches ${ }^{10}$. In the present study, we performed reverse transcription polymerase chain reaction (RT-PCR) to measure caspy 2 expression at various developmental stages. The results revealed that caspy 2 was expressed from $18 \mathrm{~h}$ postfertilization (hpf) (Fig. 5a). Consistent with this, expression of the caspy 2 protein in the whole embryo was observed from 24 hpf, and was markedly induced at 5-7 days postfertilization (dpf) (Fig. 5b). To better understand the spatial pattern of caspy 2 expression throughout zebrafish development, we performed whole-mount in situ hybridization. Consistent with previous research ${ }^{10}$, caspy 2 mRNA was detected in the mouth and pharyngeal arches from $48 \mathrm{hpf}$ (black arrowheads and white arrowheads, respectively, in Fig. 5c). Interestingly, during this time, caspy 2 expression was clearly visible in the developing gut (red arrowheads in Fig. 5c). Moreover, in adult zebrafish, substantial caspy 2 mRNA expression was detected in the gills and gut (Fig. 5d), which was significantly stronger than that in the spleen, kidney, and liver. These data demonstrated that caspy 2 is preferentially expressed in zebrafish intestinal sites, suggesting that caspy 2 might play a role in gut defenses.

In addition, we investigated the effect of caspy 2 on zebrafish development. A morpholino oligonucleotide to block caspy 2 expression was designed and injected into the embryos at the one-cell stage (Supplementary Fig. 9a, b). Immunoblotting showed that this morpholino oligonucleotide (caspy2-MO) effectively knocked down caspy 2 up to and including $7 \mathrm{dpf}$ (Supplementary Fig. 9c), and confirmed that the controlmorpholino oligonucleotide (control-MO) containing five base mismatches had no effect on caspy2 expression (Supplementary
Fig. 9c). The caspy2 morphants displayed no phenotypic abnormalities, such as general tissue loss, cell death in the head, a curled body axis, and pericardial edema (Supplementary Fig. 9d), indicating that caspy 2 knockdown did not alter zebrafish embryonic development.

Caspy2 is critical for gut inflammation and antibacterial defenses. Given that the noncanonical inflammasome is critical in combating infection by enteric bacterial pathogens at the intestinal mucosal surface ${ }^{3,16}$, we first assessed the expression of caspy2 in zebrafish larvae at $120 \mathrm{hpf}$ in response to bacterial infection by immersion (Supplementary Fig. 9a). The induction of caspy2 expression and cleavage of pro-caspy2, as determined by immunoblotting of whole zebrafish larvae extracts using an antibody against p20, were detected during 0909I E. piscicida infection, but not with wild-type E. piscicida (EIB202) infection (Fig. 6a). These results demonstrate that caspy 2 activation is significantly induced during bacterial infection in vivo, indicating its involvement in zebrafish immune defenses.

To analyze the in vivo role of caspy2 in promoting intestinal immune defenses, we performed a zebrafish larvae immersion infection procedure (Supplementary Fig. 9a). Following infection with $10^{5}$ colony forming units (CFU)/ml of 0909I E. piscicida, caspy2-MO larvae rapidly succumbed, whereas the control-MO larvae were unaffected (Fig. 6b). In agreement with a role for caspy2 in gut antibacterial defenses, caspy2-MO larvae had significantly higher gut pathogen loads (Fig. 6c, d). At early time points, initial gut colonization by 0909I E. piscicida appeared similar in caspy2-MO and control-MO larvae (Fig. 6c, d). However, by $24 \mathrm{hpi}$, it was dramatically enhanced in the former, 
a

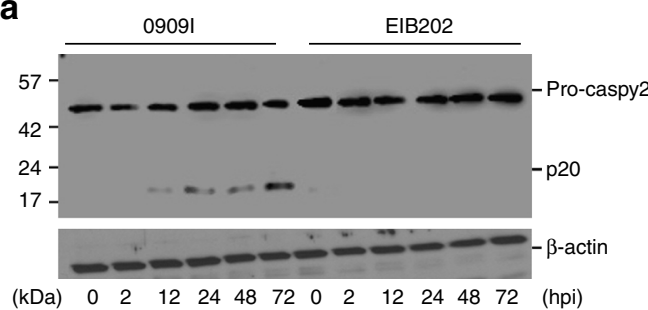

b

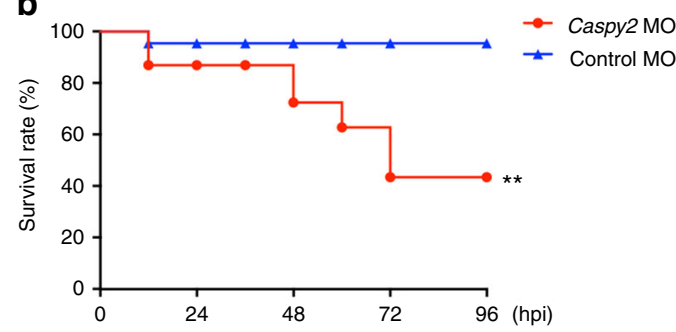

c

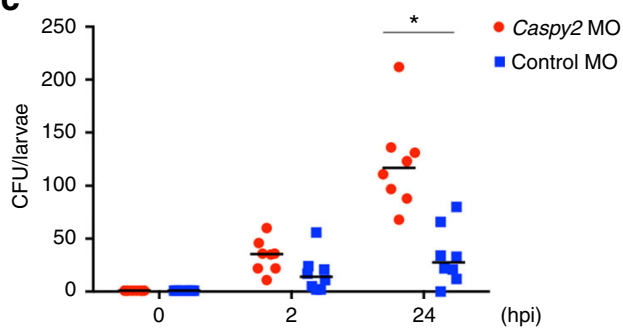

d
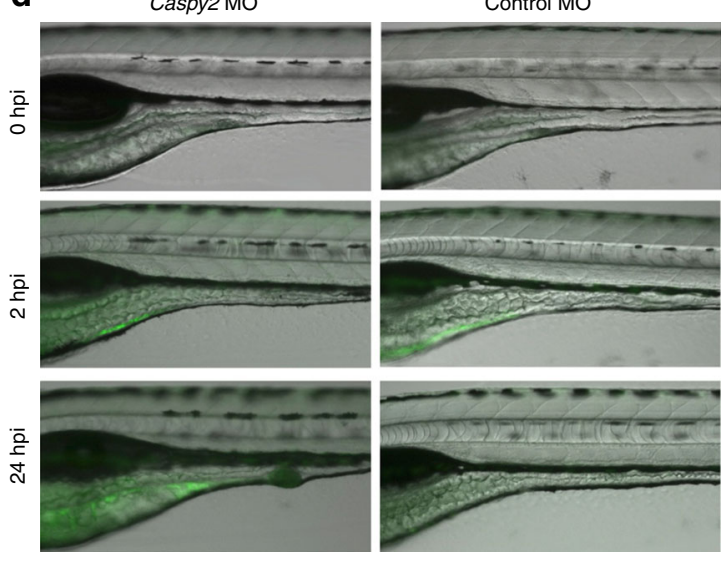

e

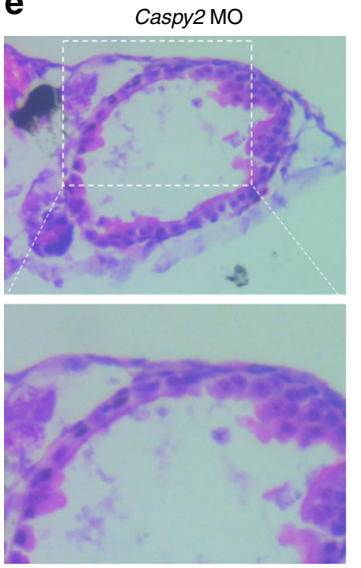

Control MO
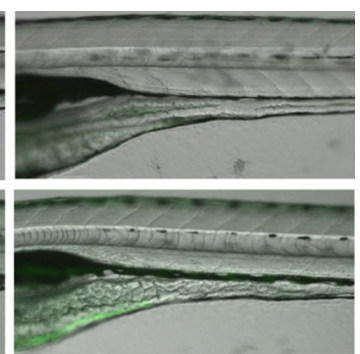

Control MO
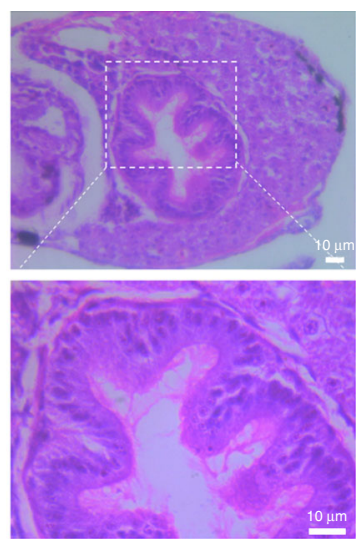

Fig. 6 Caspy2 restricts bacterial colonization of the zebrafish gut in vivo. a-e Control-morpholino oligonucleotide (MO) or caspy2-MO zebrafish larvae at 5 days postfertilization (dpf) were infected by immersion with $10^{5}$ colony forming units (CFU)/ml of 0909l E. piscicida. a Zebrafish larvae ( 5 dpf) were infected by immersion with wild-type (EIB202) or 0909I E. piscicida. Immunoblotting analysis of caspy2 levels at the indicated post-infection time points. $\mathbf{b}$ Survival of zebrafish larvae was monitored for 4 days. ( $n=60$ for control-MO, $n=60$ for caspy2-MO). Results are representative of at least three independent experiments, ${ }^{\star \star} p<0.01$ (log-rank). c Zebrafish larvae were collected at the indicated postinfection time points, and homogenates were made for CFU counts. Each symbol represents the average counts of five larvae ( $n=100$ for control-MO, $n=100$ for caspy2-MO). Results are representative of at least three independent experiments. ${ }^{\star} p<0.05$ (ANOVA). d Images of infected zebrafish larvae. Green fluorescent protein (GFP)-0909I E. piscicida. e Representative images of hematoxylin and eosin (H\&E) staining of gut sections from 0909l-infected control-MO or caspy2-MO zebrafish larvae. Square frame, intestinal wall morphology; Scale bar, $10 \mu \mathrm{m}$

but not the latter (Fig. 6c, d). Furthermore, unlike the controlMO larvae, caspy2-MO larvae exhibited prominent histopathological signs of gut inflammation after infection with 0909I $E$. piscicida (Fig. 6e). Thus, our results suggest that fish noncanonical inflammasome activation is important for bacterial clearance, playing a hitherto unrecognized role in the innate immunity of lower vertebrates.

Caspy2 is required for lethal sepsis. The predominant model for the experimental study of sepsis is the mouse, which is initiated by the administration of LPS or via pathogens infection ${ }^{17-20}$. Zebrafish embryos display many of the key pathophysiological features seen in human patients with sepsis, including dysregulated inflammatory responses (cytokine storm), tachycardia, and endothelial leakage through E. coli infection ${ }^{20}$. The noncanonical inflammasome processes was critical for septic shock in mammals ${ }^{21,22}$. In this study, we assessed the role of caspy2 in a zebrafish larval model of lethal sepsis ${ }^{23}$ by administering a lethal dose of LPS by immersion to induce lethality in $5 \mathrm{dpf}$ larvae. Control-MO larvae exposed by immersion to a lethal dose of LPS died rapidly, whereas the survival rate was higher among caspy2-
MO larvae given the same treatment (Supplementary Fig. 10a). Consistent with the morphology observed during 0909I E. piscicida infection, LPS-treated zebrafish displayed marked pericardial edema, and erosion of the tail fin and body axis in control-MO larvae after 24 hpi (Supplementary Fig. 10b). Moreover, the significant upregulation of IL- $1 \beta$, TNF- $\alpha$, IL-6, IL-8, IL-10, and IFN$\gamma$ in control-MO larvae was also observed at $12 \mathrm{hpi}$, compared with that in caspy2-MO larvae treated with LPS (Supplementary Fig. 10c). Taken together, these results indicated that activation of the noncanonical inflammasome in vivo by LPS causes rapid mortality via the noncanonical inflammasome pathway in zebrafish.

\section{Discussion}

Most previous studies concerning the assembly of the noncanonical inflammasome during inflammatory responses have focused on the functions of caspase- $4 / 5$ or caspase- 11 in mammalian cells ${ }^{24,25}$. In mouse macrophages, noncanonical inflammasomes are assembled by direct binding of LPS through the CARD domain of procaspase-11 $1{ }^{6,26}$. Caspase- 4 and caspase- 5 are the human counterparts of mouse caspase-11, which were 
also found to activate the noncanonical inflammasome in human myeloid cells ${ }^{21,22}$. Similar to the noncanonical inflammasome assembly in mice, procaspase- 4 and/or procaspase- 5 in human cells interact directly with LPS, and this interaction is mediated by binding of the CARD domain ${ }^{27-29}$. In this study, we revealed that zebrafish caspy 2 shows a caspase-5-like activity that is critical for noncanonical inflammasome activation in nonmyeloid cells (Fig. 1). However, unlike the CARD domain as found at the $\mathrm{N}$-terminal of caspase-4/5/11 in mammals, the $\mathrm{N}$-terminal domain of caspy 2 is most homologous to the pyrin domain containing NOD-family protein ${ }^{10}$. Interestingly, our data found that the pyrin domain of caspy 2 is critical in mediating intracellular LPS binding, resulting in caspy2 oligomerization and activating the noncanonical inflammasome in zebrafish (Supplementary Fig. 8). Both CARDs and PYDs belong to the death domain superfamily of signaling domains, which also includes the death-effector domains ${ }^{30,31}$. Thus, our findings reveal an unexpected concept for the evolution of pattern recognition in the death domain superfamily-mediated intracellular LPS-sensing pathway in vertebrate innate immunity (Supplementary Fig. 8).

In mammals, pyroptosis is considered to comprise caspase-1 and/or caspase-11 (caspase-4/5)-mediated cell death in response to certain bacterial insults $2,3,7,32$. Caspases selectively cleave the central linker in gasdermin family proteins, releasing and negating autoinhibition of the gasdermin- $\mathrm{N}$ domain, resulting in pyroptosis via its pore-forming activity ${ }^{7,33}$. Although we observed that caspy 2 contributes to pyroptosis of zebrafish fibroblasts in response to both cytosolic LPS and bacterial infection (Fig. 3), it remains unknown whether this enzyme cleaves a substrate to induce pyroptosis, as in mammalian cells. Previous data have already revealed that the active $\mathrm{N}$-terminal domain of the only two gasdermin family proteins identified in zebrafish, dfna5a and dfna5b, exert pore-forming effects ${ }^{33}$. Recently, zebrafish dfna5a (GSDME1) has been shown to possess a caspase-3 cleavage motif and induce pyroptosis following administration of chemotherapy drugs, although the role of dfna5b (GSDME2) remains unknown $^{33}$. Thus, we speculated that dfna5a or dfna5b, or an asyet-unidentified gasdermin family protein, fulfills the "executioner" function in fish, similar to that observed in mammals.

When caspase-4/5/11 activation by detecting cytoplasmic LPS, they trigger pyroptotic cell death and caspase- 1 dependent IL- $1 \beta$ production $^{34}$. In zebrafish, although the key components of the inflammasome complex assembly are conserved, the nod-like receptors are less well understood ${ }^{11}$. In this study, we identified the zebrafish caspy2 activation-mediated pyroptosis, both in response to cytosolic LPS and 0909I E. piscicida infection, but whether this caspy2-mediated noncanonical inflammasome activation can lead to "NLRP3 inflammasome" activation as observed in mammalian cells remain unknown. In adult zebrafish primary leukocytes, both caspy and caspy 2 can cleave IL- $1 \beta$ in response to bacterial infection ${ }^{14}$. Moreover, zebrafish ASC interacts with caspy but not with caspy2, and the oligomerized form of the conserved adapter protein ASC had been shown to recruit and activate caspy, the zebrafish functional homolog of caspase-1, forming a characteristic speck structure both in vitro ${ }^{10}$ and in vivo ${ }^{35}$. Thus, additional works still need to clarify whether the caspy 2 mediated noncanonical inflammasome activation could trigger caspy processing, is this ASC dependent? And what's the mechanism about the zebrafish IL- $1 \beta$ cleavage and secretion, is this dependent on caspy 2 or caspy?

Caspase-11 activation in intestinal epithelial cells plays an important role in the promotion of mucosal defense against enteric bacterial pathogens ${ }^{36}$, indicating that pyroptosis is not only limited to monocytes, but can also occur in nonmyeloid cells. Caspase-11 exerts proinflammatory effects and mediates gut defenses against a large variety of pathogens ${ }^{3,16,36}$. Mice deficient in caspase-11 exhibit enhanced Salmonella typhimurium colonization in the intestinal epithelium upon infection with this intracellular enteric pathogen, together with a reduction in histopathological signs of cecal inflammation ${ }^{3,4}$. Our results suggested that the noncanonical inflammasome is critical in nonmyeloid cell-mediated gut protection during 0990I E. piscicida infection in vivo. Thus, it's interesting to address the different phenotypes of mutant bacteria with that wild-type E. piscicida, which might add new findings to the molecular mechanisms in bacteria that promotes host immune recognition of replicating pathogens via noncanonical inflammasome and restricts bacterial colonization. In fish, it is generally accepted that they are exposed to substantially higher numbers of pathogens than nonaquatic vertebrates ${ }^{37}$. When a fish is exposed to pathogens during the mouth and gut opening stages, the intestine, a highly complex organ, plays a crucial role in the immune system ${ }^{38}$. However, it is not clear whether the noncanonical inflammasome exists or plays a role in fish innate immunity. In this study, we developed a bacteriazebrafish larvae immersion infection model, and demonstrate that the zebrafish caspy2 noncanonical inflammasome governs bacterial clearance and gut inflammation in vivo (Fig. 6), which paves the way for future studies to elucidate the molecular mechanisms of the noncanonical inflammasome in lower vertebrates.

In summary, we report a unique model to study the impact of noncanonical inflammasome activation in inflammation and endotoxic shock progression with clear complementarities with the mouse model. In this model, we revealed a previously unappreciated pathway of the pyrin-like domain in cytosolic LPS sensing, which mediates noncanonical inflammasome activation in zebrafish fibroblasts. Activation of the caspy2 noncanonical inflammasome plays critical role in the clearance of bacterial infection in vivo (Fig. 7). These results provide the possibility to study inflammation and pathogen responses simultaneously in a whole organism, which should lead to the possibility of genetic and chemical screenings.

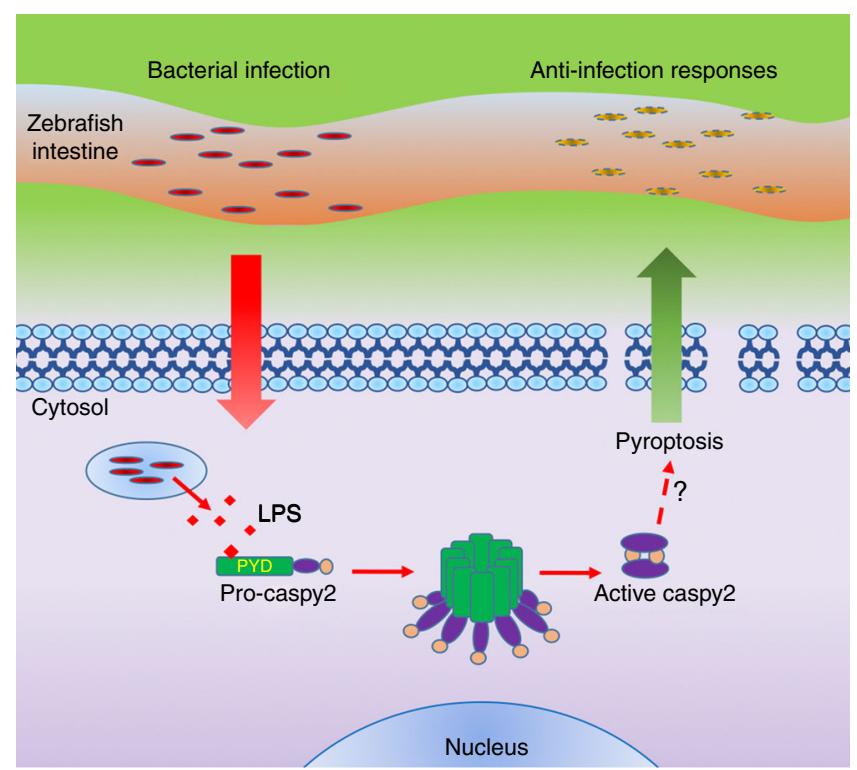

Fig. 7 Proposed mechanism for noncanonical inflammasome activation in zebrafish. Summary of the caspy2-noncanonical inflammasome activation in zebrafish illustrating the effects of antibacterial infection effects in the zebrafish intestine. LPS lipopolysaccharide; PYD pyrin death domain 


\section{Methods}

Zebrafish stocks and embryo collection. Wild-type $\mathrm{AB}$ zebrafish were maintained in the Zebrafish Core Facility of the East China University of Science and Technology (Shanghai, China) under standard conditions ${ }^{39}$. Experiments conformed to regulatory standards according to protocols approved by the Animal Care Committee of East China University of Science and Technology (approval number \#2006272). Zebrafish larvae were maintained until $10 \mathrm{dpf}$, at which time they were euthanized with an overdose of $4 \mathrm{~g} / \mathrm{L}$ buffered tricaine (MS-222, ethyl 3 aminobenzoate methanesulfonate, Sigma-Aldrich) in accordance with ethical procedures $^{39}$ (www.zfin.org).

Bacterial culture conditions. Wild-type E. piscicida (EIB202, CCTCC M208068) and 0909I E. piscicida (screened from an E. piscicda gene-defined mutant library ${ }^{40}$ ) were grown in tryptic soy broth (TSB, BD Biosciences) containing $16.7 \mu \mathrm{g} / \mathrm{mL}$ colistin at $30^{\circ} \mathrm{C}$. For infection of cells or zebrafish larvae (as described below), bacteria were grown to the stationary phase overnight in TSB at $30^{\circ} \mathrm{C}$ with aeration.

Cell transfection and infection. Mycoplasma free-ZF4 cells (ATCC CRL-2050 ), established from 1-day-old zebrafish embryos, were grown at $28^{\circ} \mathrm{C}$ in Dulbecco's modified Eagle's medium (DMEM)/F12 (Gibco) medium supplemented with $10 \%$ fetal bovine serum (FBS; Gibco) in an atmosphere containing $5 \%(\mathrm{v} / \mathrm{v}) \mathrm{CO}_{2}$. These cells were infected by replacing the culture medium with Opti-MEM (Gibco) and adding E. piscicida at a multiplicity of infection (MOI) of 50. For CTB (List Biological Laboratories) treatment, Pam3CSK4 (InvivoGen) primed cells were stimulated with $20 \mathrm{mg} / \mathrm{mL}$ CTB plus $1 \mathrm{mg} / \mathrm{mL}$ ultrapure LPS (E. coli O111:B4, InvivoGen $)^{22,41}$. For inhibitor assays, the cells were pretreated with Z-WEHDFMK (ApexBio), Z-YVAD-FMK (ApexBio), Ac-LEVD-CHO (Sigma), Z-VADFMK (Beyotime Biotechnology), Ac-DEVD-CHO (Beyotime Biotechnology), or 10 $\mathrm{mM}$ glycine (Sigma), respectively, for $30 \mathrm{~min}$ before being exposed to E. piscicida at an MOI of 50 .

Cell death and LDH release measurement. To examine the morphological changes during cell death, cells were treated as indicated in 12-well plates and images were captured. PI $(5 \mathrm{ng} / \mathrm{mL})$ was added to the medium as an indicator of cell membrane integrity. Static bright-field images of pyroptotic cells were captured using an Olympus IX71 microscope at room temperature and processed using Image J software ${ }^{42}$

Aliquots of cellular supernatants were transferred into 96-well plates (round bottom) and centrifuged at $1000 \times g$ for $5 \mathrm{~min}$. The supernatants were transferred to another 96-well plate (flat bottom), and the plate was subjected to the cytotoxicity assay using a CytoTox 96 assay kit (G1780, Promega, Madison, WI, USA) according to the manufacturer's protocol. Each sample was tested in triplicate. Cytotoxicity was normalized to Triton X-100 treatment ( $100 \%$ of control), and $\mathrm{LDH}$ release from uninfected/untreated cells was used for background subtraction.

Caspase substrate cleavage assay. ZF4 cells were lysed with caspase assay buffer (50 mM HEPES, $100 \mathrm{mM} \mathrm{NaCl}, 0.1 \%$ CHAPS, $10 \mathrm{mM}$ DTT, $1 \mathrm{mM}$ EDTA, and $10 \%$ glycerol, $\mathrm{pH} 7.4$ ) and centrifuged at $21,000 \times \mathrm{g}$ for $2 \mathrm{~min}$. To analyze of caspase5 -like activity, cell lysates or purified caspy 2 were mixed with Ac-WEHD-AFC (BioVision) in a black 96-well plate (Costar), and the resulting fluorescence was measured using a SpectraMax M2 microplate reader (excitation at $400 \mathrm{~nm}$ and emission at $505 \mathrm{~nm}$, Molecular Devices). Substrate cleavage was evident as an increase in fluorescence after $1 \mathrm{~h}$. The activity of other caspases was assayed according to the substrate manufacturer's instructions (Beyotime Biotechnology). Briefly, chromogenic substrates of human caspase-1 (Ac-YVAD-pNA), caspase-2 (Ac-VDQQD-pNA), caspase-3/7 (Ac-DEVD-pNA), caspase-4 (Ac-LEVD-pNA), caspase-8 (Ac-IETD-pNA), and caspase-9 (Ac-LEHD-pNA) were incubated with cell lysates or purified caspy 2 at a final concentration of $200 \mathrm{mM}$. Caspase activity was then determined by measuring changes in absorbance at $405 \mathrm{~nm}$ at 5 -min intervals caused by the presence of free pNA hydrolyzed from the substrates.

Generation of KO cells using CRISPR/Cas9. A CRISPR/Cas9 gRNA expression vector (pSpCas9(BB)-2A-GFP, \#48138) was obtained from Addgene. The caspy2KO target sequences were 5'-GGCGTCGAACCCATTCCTCG-3' and 5'-GAATA AGGACCGTCAGGAT-3'. To generate caspy2-KO ZF4 cell lines, DNA-In CRISPR Transfection Reagent (MTI-GlobalStem) was used to transfect the plasmids. Two to three days later, GFP-positive cells were sorted for culture by flow cytometry, and caspy2-KO clones were identified by immunoblotting with rabbitanti-caspy2 antibodies (1:1000; custom-made; Genscript).

HeLa cell reconstitution experiments. Mycoplasma free-wild-type, CASP4 ${ }^{-1-}$, and $\mathrm{GSDMD}^{-1-} \mathrm{HeLa}$ cells were grown at $37^{\circ} \mathrm{C}$ in DMEM supplemented with $10 \% \mathrm{FBS}$ in an atmosphere containing $5 \%(\mathrm{v} / \mathrm{v}) \mathrm{CO}_{2}$. CASP4 ${ }^{-/-} \mathrm{HeLa}$ cells were transduced with pCDH expression plasmids for caspy2, caspy2 (C296A), or caspy2 $(\triangle \mathrm{PYD})$. After 3 days, puromycin-resistant transduced cells were collected. Indicated cells were primed with Pam3CSK 4 for $4 \mathrm{~h}$, and stimulated with CTB plus LPS for $12 \mathrm{~h}$. Levels of caspy 2 proteins were determined by immunoblotting. CASP $4^{-1-}$
HeLa cells were transduced with pCDH expression plasmids for HA-tagged caspase-11, caspase-11 (C254S), or caspase-11 ( $\triangle \mathrm{CARD})$. After 3 days, puromycinresistant transduced cells were collected. Indicated cells were primed with Pam3CSK 4 for $4 \mathrm{~h}$, and stimulated with CTB plus LPS for $12 \mathrm{~h}$. Levels of caspase11 proteins were determined by immunoblotting using rabbit-anti-HA antibodies (1:5000; GeneTex).

Purification of proteins. Mycoplasma free-HEK293T cells were plated in a 100$\mathrm{mm}$ Petri dish $24 \mathrm{~h}$ before transfection. Cells were then cotransfected with pCDH expressed HA-tagged caspy2, caspy, caspase-1, or caspase-11. Cells were collected and lysed in a buffer containing $20 \mathrm{mM}$ Tris, $100 \mathrm{mM} \mathrm{KCl,} 0.1 \% \mathrm{NP}-40,1 \mathrm{mM}$ EDTA, $1 \mathrm{ml}$ EDTA, $10 \%$ glycerol, $10 \mathrm{mM}$ tetrasodium pyrophosphate and protease inhibitor cocktail (Roche Molecular Biochemicals) for $30 \mathrm{~min}$. The lysates were divided and incubated with Pierce Anti-HA magnetic beads (Thermo Fisher Scientific), $6 \mathrm{~h}$ later, the HA beads were washed three times with cell lysis and wash buffer (20 mM Tris, $150 \mathrm{mM} \mathrm{KCl,} \mathrm{0.5 \%} \mathrm{NP-40,} 1$ mM EDTA, 1 ml EDTA, 10\% glycerol, $10 \mathrm{mM}$ tetrasodium pyrophosphate and protease inhibitor cocktail). The HA-tagged proteins were then eluted using an HA peptide (Apexbio) according to the manufacturer's protocol.

LPS pulldown assay. Biotinylated-ultrapure LPS and Biotinylated-Pam3CSK4 from E. coli O111:B4 (InvivoGen) were incubated with Pierce ${ }^{m}$ Streptavidin Magnetic Beads (Thermo Scientific) for $6 \mathrm{~h}$. The beads were then centrifuged at $800 \times g$ for $1 \mathrm{~min}$ and the supernatant was removed. One milliliter of purified HA-tagged caspy2, caspy2 (C296A), caspy2 ( $\triangle \mathrm{PYD})$, caspy2 PYD, caspy, or caspy PYD protein in HEK293T cells was mixed with LPS or Pam3CSK4-bound beads at $4{ }^{\circ} \mathrm{C}$ overnight, after which, the beads were again centrifuged at $800 \times g$ for $1 \mathrm{~min}$ and the supernatant was removed. After three washes with PBST (phosphate buffered saline containing $0.05 \%$ Tween 20 ), the beads were boiled with sample loading buffer. The precipitates were subjected to SDS-PAGE and subsequently transferred to polyvinylidene difuoride (PVDF) membranes by electroblotting.

ELISA-based LPS binding assay. The LPS-binding activities of purified HAtagged caspy2, caspy2 (C296A), caspy2 ( $\triangle$ PYD), caspy2 PYD, caspy, caspy PYD, caspase-1, and caspase-11 proteins in HEK293T cells were measured by ELISA. Briefly, a 96-well microtiter plate (Costar) was coated with $20 \mathrm{mg}$ E. coli O111:B4 LPS (Sigma-Aldrich) in $100 \mu$ l of carbonate-bicarbonate buffer ( $\mathrm{pH} 9.6$ ) per well, and incubated at $4{ }^{\circ} \mathrm{C}$ overnight. The wells were then washed four times with PBST (containing $0.05 \%$ Tween 20 ) and blocked with $200 \mu$ l of PBS containing $3 \%$ bovine serum albumin at $37^{\circ} \mathrm{C}$ for $1 \mathrm{~h}$. The plate was thoroughly washed, and $100-\mu \mathrm{l}$ twofold serial dilutions of purified protein were added to each well and incubated at room temperature for $2 \mathrm{~h}$. The plate was again thoroughly washed, and a mouse anti-HA tag monoclonal antibody (diluted 1:2000, Abcam) was added and incubated at room temperature for $1 \mathrm{~h}$ to detect the bound purified proteins.

Caspy2 foci staining and caspy2 oligomer cross-linking. ZF4 cells were seeded on coverslips at a density of $2 \times 10^{5}$ cells per well in 24 -well plates and cultured overnight. After infection with indicated bacterial strains, cells were washed with PBS and fixed with $4 \%$ paraformaldehyde for $15 \mathrm{~min}$, permeabilized with $0.1 \%$ Triton X-100 for $5 \mathrm{~min}$, and blocked with 10\% FBS in PBS for $20 \mathrm{~min}$. Cells were then incubated with anti-caspy 2 antibodies (1:1000; custom-made; Genscript), followed by goat antirabbit $\operatorname{IgG}(\mathrm{H}+\mathrm{L})$ secondary antibody, oregon green $488(\mathrm{O}$ 11038, Thermo Fisher Scientific) and 2-(4-amidinophenyl)-1H-indole-6-carboxamidine (C1005, Beyotime Biotechnology). Images were acquired using a Leica DMI3000B inverted fluorescence microscope.

For caspy2 oligomer cross-linking, ZF4 cells were plated on 12-well plates and stimulated as indicated. Cells were lysed with PBS buffer containing $0.5 \%$ Triton X100 , and the lysates were centrifuged at $6797 \times g$ for $15 \mathrm{~min}$ at $4{ }^{\circ} \mathrm{C}$. Supernatants were transferred to new tubes (Soluble fractions). The Triton X-100-insoluble pellets were washed with PBS twice and then suspended in $200 \mu \mathrm{PBS}$. The pellets were then cross-linked at room temperature for $30 \mathrm{~min}$ by adding $4 \mathrm{mM}$ dextran sulfate sodium. The cross-linked pellets were centrifuged at $6797 \times g$ for $15 \mathrm{~min}$ and dissolved directly in SDS sample buffer.

Blue native PAGE. Blue native gel electrophoresis was performed using the Bis-Tris native PAGE system as previously described ${ }^{6,43}$. ZF4 cells were plated on 12 -well plates and stimulated as indicated. Cells were washed once with cold PBS and then lysed in ice-cold native lysis buffer $(20 \mathrm{mM}$ Bis-Tris, $500 \mathrm{mM} \varepsilon$ aminocaproic acid, $20 \mathrm{mM} \mathrm{NaCl}, 10 \%(\mathrm{w} / \mathrm{v})$ glycerol, $0.5 \%$ digitonin, $0.5 \mathrm{mM}$ $\mathrm{Na}_{3} \mathrm{VO}_{4}, 1 \mathrm{mM}$ PMSF, $0.5 \mathrm{mM} \mathrm{NaF}, 1 \times$ EDTA-free Roche protease inhibitor cocktail, $\mathrm{pH} 7.0$ ) for $15 \mathrm{~min}$ on ice. Lysates were clarified by centrifugation at $20,000 \times g$ for $30 \mathrm{~min}$ at $4{ }^{\circ} \mathrm{C}$ and analyzed without further purification steps. Cell lysates were equalized after quantification of total protein using the BCA protein assay (Pierce), and then separated by $4-12 \%$ blue native PAGE. Native gels were incubated in $10 \%$ SDS solution for 5 min before transfer to PVDF membranes (Millipore), followed by conventional western blotting. 
Morpholino design and analysis. An morpholino oligonucleotide (Gene Tools) was designed to target a site in caspy 2 to block its translation (5'-CTGGGTAAT ATCCTCCATTTTCTGT-3'), and a corresponding 5-base mismatch oligonucleotide was used as a control (5'-CTGGcTAATATgCTgCATTTTgTcT-3'). Embryos were injected as described previously ${ }^{44}$. MOs were used at $0.5 \mathrm{ng}$ per embryo, and the results of experiments using three batches of embryos, performed and analyzed independently, are shown. Knockdown of caspy2 expression was verified by immunoblotting with rabbit-anti-caspy2 antibodies (1:1000; custom-made; Genscript).

Immunoblotting. Lysates were subjected to SDS-PAGE before being transferred to PVDF membranes (Millipore) by electroblotting. For immunoblotting, the rabbitanti-caspy2 antibody (1:1000; custom-made; Genscript), or rabbit-anti-HA antibody (1:5000; GeneTex) were used. The same amounts of lysates were leaded on each gel and probed with anti- $\beta$-actin antibodies (1:5000; Abcam) as a loading control.

Whole-mount in situ hybridization. For whole-mount in situ hybridization, digoxigenin-labeled caspy2 antisense RNA probes were synthesized from fulllength cDNA sequences using an in vitro transcription kit (Promega). In situ hybridization and development of whole-mount zebrafish embryos were performed as described previously ${ }^{10}$.

Bacterial immersion infection model. For immersion infection of zebrafish larvae, bacteria were prepared as described in the Bacterial Culture Conditions subsection. Larvae at $5 \mathrm{dpf}$ were randomly divided and immersed in PBS or PBS containing $10^{5} \mathrm{CFU} / \mathrm{ml}$ 0909I E. piscicida for $2 \mathrm{~h}$, before being allowed to recover for $15 \mathrm{~min}$ in a petri dish containing fresh E3 medium. Subsequently, they were transferred to $10-\mathrm{cm}$ dishes, with approximately 50 larvae in $15 \mathrm{ml}$ of E3 medium per dish, and incubated at $28^{\circ} \mathrm{C}$. The mortality rate and bacterial colonization were then observed at different time points. For histopathology, embryos were injected with the caspy2- or control-MO at the one-cell stage, and fixed in $4 \%$ paraformaldehyde at $72 \mathrm{hpi}$. Whole larvae were dissected and tissues fixed in formalin and embedded in paraffin using standard methods ${ }^{39}$. Tissue sections were evaluated microscopically for signs of inflammation following hematoxylin and eosin staining ${ }^{39}$.

Lethal sepsis model. Zebrafish larvae at $5 \mathrm{dpf}$ were randomly divided and immersed in $100 \mu \mathrm{g} / \mathrm{ml}$ E. coli O111:B4 LPS (Sigma) for $2 \mathrm{~h}$, before being allowed to recover for $15 \mathrm{~min}$ in a petri dish containing fresh E3 medium. Subsequently, they were transferred to $10-\mathrm{cm}$ dishes, with approximately 50 larvae in $15 \mathrm{ml}$ of E3 medium per dish, and incubated at $28^{\circ} \mathrm{C}$. Survival rates were calculated at the indicated time points. The RNA of infected zebrafish larvae was extracted using an RNA isolation kit (Tiangen, Beijing, China). One microgram of each RNA sample was used for cDNA synthesis with the FastKing One Step RT-PCR Kit (Tiangen) and quantitative real-time PCR (qPCR) was performed on an FTC-200 detector (Funglyn Biotech, Shanghai, China) by using the SuperReal PreMix Plus (SYBR Green) (Tiangen). The expression level of the genes ecoding IL-1 $\beta$, TNF- $\alpha$, IL- 6 , IL-8, IL-10, and IFN- $\gamma$ (Supplementary Table 1) were assessed for three biological replicates, and the data for each sample were expressed relative to the expression level of the gene encoding beta-actin gene by using the $2^{-\Delta \Delta C T}$ method ${ }^{39}$.

Statistical analysis. Statistical analysis was performed using GraphPad Prism (GraphPad Software). All data are representative of at least three independent experiments and are presented as means $\pm \mathrm{SD}$. Two-group comparisons were made using Student's $t$ test, and one-way analysis of variance was used to assess differences among multiple groups. Differences in larvae survival were assessed using the log-rank (Mantel-Cox) test. $p$ Values less than 0.05 were considered to indicate statistical significance $\left({ }^{*} p<0.05,{ }^{* *} p<0.01\right)$.

Data availability. All relevant data are available from the authors on request and/ or are included with the manuscript (as figure source data or supplementary information files).

Received: 29 September 2017 Accepted: 17 April 2018

Published online: 03 August 2018

\section{References}

1. Crowley, S.M., Vallance, B.A., \& Knodler, L.A. Noncanonical inflammasomes: antimicrobial defense that does not play by the rules. Cell Microbiol. 19, e12730 (2017)

2. Aachoui, Y. et al. Caspase-11 protects against bacteria that escape the vacuole. Science 339, 975-978 (2013).
3. Knodler, L. A. et al. Noncanonical inflammasome activation of caspase-4/ caspase-11 mediates epithelial defenses against enteric bacterial pathogens. Cell Host Microbe 16, 249-256 (2014).

4. Thurston, T. L. et al. Growth inhibition of cytosolic Salmonella by caspase-1 and caspase-11 precedes host cell death. Nat. Commun. 7, 13292 (2016).

5. Shi, J., Gao, W. \& Shao, F. Pyroptosis: gasdermin-mediated programmed necrotic cell death. Trends Biochem. Sci. 42, 245-254 (2017).

6. Shi, J. et al. Inflammatory caspases are innate immune receptors for intracellular LPS. Nature 514, 187-192 (2014).

7. Shi, J. et al. Cleavage of GSDMD by inflammatory caspases determines pyroptotic cell death. Nature 526, 660-665 (2015).

8. Torraca, V., Mostowy, S. Zebrafish infection: from pathogenesis to cell biology. Trends Cell Biol. 28, 143-156 (2018).

9. Sakamaki, K. \& Satou, Y. Caspases: evolutionary aspects of their functions in vertebrates. J. Fish Biol. 74, 727-753 (2009).

10. Masumoto, J. et al. Caspy, a zebrafish caspase, activated by ASC oligomerization is required for pharyngeal arch development. J. Biol. Chem. 278, 4268-4276 (2003).

11. Li, Y., Li, Y., Cao, X., Jin, X. \& Jin, T. Pattern recognition receptors in zebrafish provide functional and evolutionary insight into innate immune signaling pathways. Cell. Mol. Immunol. 14, 80-89 (2017).

12. Mostowy, S. et al. The zebrafish as a new model for the in vivo study of Shigella flexneri interaction with phagocytes and bacterial autophagy. PLoS Pathog. 9, e1003588 (2013).

13. Vincent, W. J. B., Freisinger, C. M., Lam, P., Huttenlocher, A. \& Sauer, J. D. Macrophages mediate flagellin induced inflammasome activation and host defense in zebrafish. Cell Microbiol. 18, 591-604 (2016).

14. Vojtech, L. N., Scharping, N., Woodson, J. C., \& Hansen, J. D. Roles of inflammatory caspases during processing of zebrafish interleukin- $1 \beta$ in Francisella noatunensis infection. Infect. Immun. 80, 2878-2885 (2012).

15. Liu, L. et al. A novel strategy for tumour therapy combining cell apoptosis and active immunity induced by caspy2, a zebrafish caspase. J. Cell Mol. Med. 13, 2271-2281 (2009).

16. Pallett, M. A. et al. Bacterial virulence factor inhibits caspase- $4 / 11$ activation in intestinal epithelial cells. Mucosal Immunol. 10, 602-612 (2017).

17. Schildberger, A., Rossmanith, E., Weber, V. \& Falkenhagen, D. Monitoring of endothelial cell activation in experimental sepsis with a two-step cell culture model. Innate Immun. 16, 278-287 (2010).

18. Pathak, E. \& Mayeux, P. R. In vitro model of sepsis-induced renal epithelial reactive nitrogen species generation. Toxicol. Sci. 115, 475-481 (2010).

19. Seok, J. et al. Genomic responses in mouse models poorly mimic human inflammatory diseases. Proc. Natl Acad. Sci. USA 110, 3507-3512 (2013).

20. Barber, A.E. Fleming, B.A., Mulvey, M.A., Similar lethal strains of extraintestinal pathogenic Escherichia coli trigger markedly diverse host responses in a zebrafish model of sepsis. Msphere 1, e00062-16 (2016).

21. Hagar, J. A., Powell, D. A., Aachoui, Y., Ernst, R. K. \& Miao, E. A. Cytoplasmic LPS activates caspase-11: implications in TLR4-independent endotoxic shock. Science 341, 1250-3 (2013).

22. Kayagaki, N. et al. Noncanonical inflammasome activation by intracellular LPS independent of TLR4. Science 341, 1246-9 (2013).

23. Na, Y. R. et al. Consistent inhibition of cyclooxygenase drives macrophages toward the inflammatory phenotype. PLoS One 10, e0118203 (2015).

24. Jorgensen, I., Rayamajhi, M. \& Miao, E. A. Programmed cell death as a defense against infection. Nat. Rev. Immunol. 17, 151-164 (2017).

25. Kesavardhana, S. \& Kanneganti, T. D. Mechanisms governing inflammasome activation, assembly and pyroptosis induction. Int. Immunol. 29, 201-210 (2017).

26. Yang, J., Zhao, Y. \& Shao, F. Noncanonical activation of inflammatory caspases by cytosolic LPS in innate immunity. Curr. Opin. Immunol. 32, 78-83 (2015).

27. Casson, C. N. et al. Human caspase-4 mediates noncanonical inflammasome activation against gram-negative bacterial pathogens. Proc. Natl Acad. Sci. USA 112, 6688-6693 (2015).

28. Viganò, E. et al. Human caspase- 4 and caspase- 5 regulate the one-step noncanonical inflammasome activation in monocytes. Nat. Commun. 6, 8761 (2015).

29. Wacker, M. A., Teghanemt, A., Weiss, J. P. \& Barker, J. H. High-affinity caspase-4 binding to LPS presented as high molecular mass aggregates or in outer membrane vesicles. Innate Immun. 23, 336-44 (2017).

30. Park, H. H. et al. The death domain superfamily in intracellular signaling of apoptosis and inflammation. Annu. Rev. Immunol. 25, 561-586 (2007).

31. Kersse, K., Verspurten, J., Vanden Berghe, T. \& Vandenabeele, P. The deathfold superfamily of homotypic interaction motifs. Trends Biochem. Sci. 36, 541-552 (2011).

32. Broz, P. et al. Caspase-11 increases susceptibility to Salmonella infection in the absence of caspase-1. Nature 490, 288-291 (2012).

33. Wang, Y. et al. Chemotherapy drugs induce pyroptosis through caspase-3 cleavage of a gasdermin. Nature 547, 99-103 (2017). 
34. Baker, P. J. et al. NLRP3 inflammasome activation downstream of cytoplasmic LPS recognition by both caspase-4 and caspase-5. Eur. J. Immunol. 45, 2918-2926 (2015).

35. Kuri, P. et al. Dynamics of in vivo ASC speck formation. J. Cell Biol. 216, 2891-2909 (2017).

36. Zmora, N., Levy, M., Pevsner-Fischer, M., \& Elinav, E. Inflammasomes and intestinal inflammation. Mucosal Immunol. 10, 865 (2017).

37. Fuhrman, J. A. Marine viruses and their biogeochemical and ecological effects. Nature 399, 541e548 (1999).

38. Martin, S. A. M., Dehler, C. E. \& Krol, E. Transcriptomic responses in the fish intestine. Dev. Comp. Immunol. 64, 103-117 (2016).

39. Westerfield, M. The Zebrafish Book. A Guide for the Laboratory Use of Zebrafish (Danio rerio). 4th ed., (University of Oregon Press, Eugene, 2000).

40. Yang, G. et al. Time-resolved transposon insertion sequencing reveals genome-wide fitness dynamics during infection. mBio 8, 5e01581-17 (2017).

41. Yang, D., He, Y., Muñoz-Planillo, R., Liu, Q. \& Nunez, G. Caspase-11 requires the pannexin-1 channel and the purinergic $\mathrm{P} 2 \mathrm{X} 7$ pore to mediate pyroptosis and endotoxic shock. Immunity 43, 923-932 (2015).

42. Schneider, C. A., Rasband, W. S. \& Eliceiri, K. W. NIH Image to ImageJ: 25 years of image analysis. Nat. Methods 9, 671-675 (2012).

43. He, Y., Zeng, M. Y., Yang, D., Motro, B. \& Nunez, G. Nek7 is an essential mediator of NLRP3 activation downstream of potassium efflux. Nature 530, 354-357 (2016).

44. Flynt, A. S., Rao, M. \& Patton, J. G. Blocking zebrafish microRNAs with morpholinos. Methods Mol. Biol. 1565, 59-78 (2017).

\section{Acknowledgments}

We thank Dr. Feng Shao from NIBs for sharing CASP4 $4^{-1-}$ and GSDMD ${ }^{-1-} \mathrm{HeLa}$ cells, and providing useful advice. This work was supported by the National Natural Science Foundation of China (Nos. 31472308 (Q.L.) and 31430090 (Y.Z.)) and the Fundamental Research Funds for the Central Universities (No. 222201714022 (D.Y.)). Dahai Yang was supported by the Young Elite Scientists Sponsorship Program by CAST No. 2016QNRC001, Shanghai Pujiang Program No. 16PJD020, Shanghai Chenguang Program No. 16CG33, and Talent Program of School of Biotechnology in East China University of Science and Technology. Ruilin Zhang was supported by the National Natural Science Foundation of China (No. 31571492).

\section{Author contributions}

D.Y. and Q.L. were responsible for project conception, data analysis, and manuscript editing; D.Y. performed the majority of experiments, assisted by S.C., X.Z., Z.W., W.X., J. T., T.H., M.H., W.W., and G.Z.; X.Z. helped with the in vivo experiments. Q.W., R.Z., and Y.Z. provided expert advice and critically reviewed the manuscript. The manuscript was written by D.Y. and Q.L., who also supervised the study.

\section{Additional information}

Supplementary Information accompanies this paper at https://doi.org/10.1038/s41467018-04984-1.

Competing interests: The authors declare no competing interests.

Reprints and permission information is available online at http://npg.nature.com/ reprintsandpermissions/

Publisher's note: Springer Nature remains neutral with regard to jurisdictional claims in published maps and institutional affiliations.

\begin{abstract}
(c) (i) Open Access This article is licensed under a Creative Commons Attribution 4.0 International License, which permits use, sharing, adaptation, distribution and reproduction in any medium or format, as long as you give appropriate credit to the original author(s) and the source, provide a link to the Creative Commons license, and indicate if changes were made. The images or other third party material in this article are included in the article's Creative Commons license, unless indicated otherwise in a credit line to the material. If material is not included in the article's Creative Commons license and your intended use is not permitted by statutory regulation or exceeds the permitted use, you will need to obtain permission directly from the copyright holder. To view a copy of this license, visit http://creativecommons.org/ licenses/by/4.0/.
\end{abstract}

(C) The Author(s) 2018 